\title{
Herpes Simplex Encephalitis initially erroneously diagnosed as glioma of the cerebellum. Case report and literature review.
}

Dimitrios Panagopoulos ( $\nabla$ dimpanayop@gmail.com )

Geniko Nosokomeio Paidon Agia Sofia

loannis Tsonis

Geniko Nosokomeio Paidon Agia Sofia

Katerina Apostolopoulou

Geniko Nosokomeio Paidon Agia Sofia

Ploutarchos Karydakis

251 Hellenic Air Force General Hospital

Georgios Sfakianos

Geniko Nosokomeio Paidon Agia Sofia

Marios Themistocleous

Geniko Nosokomeio Paidon Agia Sofia

\section{Case Report}

Keywords: Herpes Encephalitis, glioma

Posted Date: February 18th, 2019

DOI: https://doi.org/10.21203/rs.2.350/v1

License: (9) (i) This work is licensed under a Creative Commons Attribution 4.0 International License. Read Full License

Version of Record: A version of this preprint was published at World Neurosurgery on September 1st, 2019. See the published version at https://doi.org/10.1016/j.wneu.2019.06.158. 


\section{Abstract}

\section{Background}

Herpes simplex Encephalitis caused by Herpes simplex has an estimated annual prevalence in the order of 1 in 250,000 to 500,000 patients and is considered to be the most usually encountered non endemic pathogenic cause of lethal encephalitis in well developed countries. There are a few cases reported in the literature in which a diagnostic dilemma between was raised between herpes simplex encephalitis and brain glioma, and a definitive diagnosis was difficult to be obtained.

\section{Case presentation}

We report the case of a five year old female with previous medical history of premature thelarche. As a part of her investigation, an MRI of the brain was performed, which recognized a space-occupying lesion of the posterior fossa. An MRS was performed which advocated over the diagnosis of glioma. She was operated on but the histopathological analysis failed to verify the imaging findings. HSV 1 was detected in CSF samples. Follow up MRI scans illustrated the progression of the disease

The accumulation of data regarding CSF sample analysis, EEG, brain biopsy and imaging findings, along with the progression of the clinical picture of our patient, verified the diagnosis of HSV encephalitis.

\section{Conclusions}

When confronted with confounding data that can pose a diagnostic dilemma between HSV encephalitis and glioma, brain biopsy and PCR of CSF samples could be able to verify the definite diagnosis. When interpreting our results, always we have to consider the evolution of the clinical picture.

\section{Background}

Herpes simplex encephalitis has an estimated annual incidence of 1 in 250,000 to 500,000 persons and is considered to be the most common sporadic lethal encephalitis in the Western world $[1,2]$. It is commonly manifested with headache, fever, and confusion, progressing over hours to days. Focal neurological findings including hemiparesis, cranial nerve deficits, visual field loss, and dysphasia, as well as focal or generalized seizures are included in the spectrum of clinical manifestations $[1,3]$.

Gliomas also demonstrate MRI findings of hypointensity on T1 images and hyperintensity on T2 images that is similar to the imaging characteristics of herpes encephalitis. The clinical features of viral encephalitis, namely headache, fever, seizures, and encephalopathy, are not pathognomonic of this entity and may be also seen in patients with high-grade gliomas [4]. In this manuscript, we describe a case of a space-occupying lesion of the posterior fossa which proved to be a manifestation of HSV encephalitis instead of glioma, as it was initially thought to be. To the best of our knowledge, the relevant existing literature is exceedingly restricted, and more specifically we have not encountered any other case description of a posterior fossa lesion mimicking HSV encephalitis lesion, which proved to be 
encephalitis, based on the evolving clinical evaluation, PCR and histopathologic analysis with specific stains.

\section{Case Presentation}

We refer to the case of a five year old girl who has a known medical history of premature thelarche (mentioned at age of 18 months). LHRH diagnostic test revealed a pre-pubertal type of response, excluding the diagnosis of premature puberty. Radiographic evaluation of bone maturity revealed a slight discrepancy between the skeletal and chronological age, in favor of the skeletal age. The patient was under endocrinological evaluation and she underwent an MRI of the region of the hypophysishypothalamus for further diagnostic investigation. No symptoms of encephalopathy / viral meningitis / encephalitis at the time of the initial MRI were clinically detected and the imaging was executed on an outpatient basis.

The anterior gland of the hypophysis did not reveal any pathologic changes regarding its configuration and shape, whereas the posterior gland was of normal size, with bright spots recognized within its substance. After the administration of paramagnetic substance, no pathologic uptake was detected from the parenchyma of the gland.

Another finding that was identified during the examination was a focal lesion with pathologic signal characteristics, detected in the white matter of the right cerebellar hemisphere, its maximum diameter measuring $23 \mathrm{~mm}$. It was characterized by the presence of a lobular margin and the presence of moderate midline shift. After contrast administration, there was no contrast uptake- due to these atypical features, MRS was recommended (Figures 1.A, 1.B, 1.C). Neither relevant medical, family, and psychosocial history including relevant genetic information were obtained, no past interventions. The relevant physical examination did not reveal anything and other significant clinical findings were not observed.

MRS revealed that in the substance of the space-occupying lesion there was increased concentration of choline and a moderate decrease in the concentration of NAA. The fractions of the measured metabolites were abnormal $(\mathrm{NAA} / \mathrm{Cr}=0,89, \mathrm{Cho} / \mathrm{Cr}=1,41$ and Cho/NAA $=1,58)$ (Figure 2). The aforementioned measurements were compatible with a low grade lesion, such as a grade I astrocytoma. The imaging evaluation was supplemented to include the entire neuraxis, but no pathologic contrast uptake across its entire length was detected. Futhermore, no pathologic signal intensity was recognized during the evaluation of the entirety of the spinal cord.

A few days after performance of the imaging testing, the patient underwent a right suboccipital craniotomy in order to access and remove the hemispheric lesion. Intraoperatively, no clearly defined space occupying lesion involving the cerebellar hemisphere was identified. There was a discrepancy between the preoperative imaging characteristics and the macroscopic intraoperative findings, so multiple specimens were send for histopathologic analysis. 
The immune-histochemical analysis revealed that there was GFAP expression to the existing astrocytes, diffuse synaptophysin expression, neurofilaments $2 \mathrm{~F} 11$ and NeuN to the existing neurons no significant expression of the mitotic labeling index Ki- 67/MIB-1 was detected, in any particular cell population. The specimens included cerebellar tissue elements without remarkable gliosis, with heterogeneous vascular congestion and focuses with moderate to severe hemorrhagic deposition and regional necrosis, without other pathognomonic findings.

No inclusions of viral origin into neural cells were recognized. Regions of mild to moderate chronic infection were elicited, without specific characteristics resembling granulomatous infection. The aforementioned findings are not conclusive of a specific histopathologic entity the possibility that these findings are compatible with being part of a nearby major lesion which is not represented to the excised and examined specimen material, cannot be excluded.

After the operation and while the patient was in the ICU, we executed a CT scan before extubation which revealed the presence of a well circumscribed region in the territory of the right cerebellar hemisphere behind the anatomic position of the fourth ventricle (Figure 3 ). This finding was thought to represent a remaining tumor remnant.

Facing this diagnostic dilemma, we decided to perform a second-look operation, in order to re-evaluate the surgical bed and the nearby field but the histopathological diagnosis remained unchanged.

Besides that, while the patient was treated in the ICU after the second operation, after the discontinuation of sedation, the patient exhibited intention tremor, slowness in the execution of voluntary movements, inability to speak and occasionally involuntary downward gas movement. Due to episode of gas attachment accompanied by hypertonia and tremor of the extremities, the patient started phenytoin as antiepileptic medication.

After the patient was transferred back to the ward, a new episode of generalized tonic-clonic seizures was reported and oxcarbazepine was added to its medication scheme. EEG was performed, without sleep deprivation, which revealed the presence of $\delta$ waves $(2-3 \mathrm{c} / \mathrm{sec})$, of intermediate to high amplitude, as well as, less frequently, the presence of $\theta$ waves (4-5c/s). The EEG diagram seems to be completely disorganized, finding that could be attributed to viral encephalitis. Besides that, the patient became febrile $\left(\mathbb{3 8}, 5^{\circ} \mathrm{C}\right)$ and there were episodes of salivation and horizontal nystagmoid eye movements. The patient was lethargic, not communicating well, with slurred speech, poor eye contact, was exhibiting decreased psychomotor activity and word comprehension difficulties.

The usual work up for evaluation (culture of blood, CSF and urine samples, $x$-ray of the chest) of the etiology of fever was negative and the diagnostic possibility of herpes encephalitis was enhanced.

Based on that data, we considered the possibility that we were treating a case of "pseudotumor», that is a lesion masquerading as tumor. We continued our diagnostic work up with the examination of blood 
samples for RNA of Poliovirus, Coxsackieviruses A and B, Echovirus and Enterovirus. The method was One Step Real Time qRT-PCR (TaqMan) (Gene 5' UTR, Human RNase P) · the end result was negative.

We also examined CSF samples in order to detect DNA of HSV 1 and 2 (method Real Time PCR (FRET). The genes to be detected were encoding UL28 (ICP18.5) and Glycoprotein B (UL27). The first PCR assay for HSV DNA was negative, but it is well established that if the sample is taken on the first or second day of illness, it could be negative and may become positive on testing of a subsequent CSF specimen [5]. Our strategy was to repeat the examination a few days later- the result was negative for HSV2 but it was positive for HSV1. Similar tests were obtained for the detection of RNA of Influenza viruses A and B and HCMV from CSF samples but the results were negative. Besides, basic CSF testing from both specimens (leukocyte count and protein) were supportive of our diagnosis, since the clinical diagnosis of HSV is essentially non-existent without elevations in these values. More specifically, leukocyte count was 120 cells / $\mu \mathrm{L}$, with mononuclear cell pleocytosis, and protein concentration $96 \mathrm{mg} / \mathrm{dl}$ (glucose level was $60 \mathrm{mg} / \mathrm{dl}$ ). Blood sample tests did not reveal any leukocytosis (white cell count was $6.300 / \mu \mathrm{L}$ ), or electrolyte imbalance. Erythrocyte sedimentation rate was $62 \mathrm{~mm} / \mathrm{hour}$, and CRP was $7.5 \mathrm{mg} / \mathrm{dL}$. Urine toxicology screening was negative.

After PCR confirmation of HSV I in CSF, we attended to verify our diagnosis via further evaluation of the received brain biopsy specimens. Before PCR, diagnosis of HSV via brain biopsy was the only method to diagnose HSV. To the best of our knowledge, it remains one of the most specific tests for diagnosis of HSV infection. Individuals may have HSV nucleic acid in their CSF without severe symptoms (i.e., Mollaret's meningitis). In our experience, HSV nucleic acid is necessary, but not sufficient to diagnose HSV encephalitis. Histopathologic analysis was supplemented by viral stain. The demonstration of the presence of HSV antigen by DFA staining (immunofluorescence) of tissue sample, examined using a fluorescence microscope, verified our diagnosis.

As the diagnosis of herpes encephalitis was established, the patient received treatment with acyclovir $(60 \mathrm{mg} / \mathrm{kg}, 350 \mathrm{mgx} 4)$. The treatment regimen was scheduled to be given for a period of three weeks and to be discontinued if PCR of CSF was negative for HSV1. Actually, this was the case for our patient.

Six months after the operation, a repeat MRI was performed in order to evaluate the progression of the disease. The patient was under medication with oxcarbazepine the initial daily dose $600 \mathrm{mg} /$ day, given twice a day and the target maintenance dose, which was achieved over two weeks, was $31 \mathrm{mg} / \mathrm{kg}$, approximately $900 \mathrm{mg} /$ day. Her cognitive and neurological condition remained unchanged. The repeat MRI revealed that gliotic tissue has appeared in the surgical resection cavity with concordant enlargement of the gyral sulci of the relevant cerebellar hemisphere. The pathologic area located in the region of the resection cavity retains similar imaging characteristics with that identified in the previous imaging that is high signal intensity on T2W and FLAIR sequences, with mild restriction of its dimensions. It also delineated the existence of a limited area with high signal intensity in the region of the right cerebellar peduncle, without enhancement after contrast administration. There is an extensive area of gliosis located to the cortex of the left fronto-parietal region of the cerebral hemisphere, with 
associated enlargement (ex vacuo) of the body, the frontal and temporal horn of the ipsilateral lateral ventricle (Figure 4.A, 4.B, 4.C) (the patient exhibits a right-sided hemiparesis, with hypertonia of her left side with increased deep tendon reflexes ipsilaterally).

The patient underwent serial repeated MRI examinations, in order to monitor the possible development of additional pathological features, but the only differentiating finding with respect to the previous images was the visualization of atrophic changes in the domain of the right cerebral peduncle, without enhancement after administration of contrast medium. The final imaging was performed 3 years after the initial radiological evaluation, which verified the aforementioned findings (Figure 5.A, 5.B, 5.C, 5.D).

EEG revealed a basic rhythm of $\theta$ waves (5-6c/sec) (discharges with rhythmic frequency) when the patient was waking, which could represent an ictal onset pattern. During all the recording period, we recognize the very frequent occurrence of paroxysmal discharges of spike-multispike wave form and of focal sharp waves that are mainly recorded from the scalp electrodes covering the fronto-temporal regions of the left cerebral hemisphere. These tend to propagate from the generating source to the relevant regions of the contralateral hemisphere and become generalized (generalized discharges). It is worthwhile to mention that spikes in the waking state (or during active sleep) provide the best localizing data.

Our basic tenet was to clarify the underlying diagnosis of our patient, given the restrictions that were imposed by the conflicting data of the imaging modalities and the histopathologic specimen analysis. We were confronted with a discrepancy between the clinical picture of our patient at presentation and her imaging characteristics from one side, and the absence of verification of our suspicion from the histopathologic analysis, from the other side.

Eventually, after our patient was operated twice, due to a suspicious finding on the immediate postoperative CT scan, the clinical evolution of the disease directed our diagnostic work-up to the exclusion or verification of encephalitis.

We need to mention that, immediately after the first operation, during our patient stay to the ICU, the imaging modality that was used to evaluate our postoperative outcome was a CT scan. As far as, based on our preoperative data and concerning that the results of the preliminary histopathologic evaluation were not diagnostic about the tissue of origin of the resected specimen, another concern was the execution of an immediate postoperative MRI. Unfortunately, our patient was hemodynamically unstable and the execution of an MRI scan was not considered to be a safe choice. We needed an examination to rule out any offending pathology in the territory of the surgical wound, which would be able to exclude any surgical complication (edema-hematoma-infarction). As soon as CT scan ruled out any major surgical complications but raised suspicion about the completeness of the resection, we had to decide either to perform an MRI as soon as possible, or to re-operate our patient (second look operation). We decided to re-operate, based on the concept that the histopathology specimen did not seem to be diagnostic and on the possibility that a new MRI could yield insufficient information, unable to help us resolve our diagnostic dilemma. 
Six months after the operation, a repeat MRI was performed in order to evaluate the progression of the disease. The patient was under medication with oxcarbazepine the initial daily dose $600 \mathrm{mg} /$ day, given twice a day and the target maintenance dose, which was achieved over two weeks, was $31 \mathrm{mg} / \mathrm{kg}$, approximately $900 \mathrm{mg} /$ day. Her cognitive and neurological condition remained unchanged. The repeat MRI revealed that gliotic tissue has appeared in the surgical resection cavity with concordant enlargement of the gyral sulci of the relevant cerebellar hemisphere. The pathologic area located in the region of the resection cavity retains similar imaging characteristics with that identified in the previous imaging that is high signal intensity on T2W and FLAIR sequences, with mild restriction of its dimensions. It also delineated the existence of a limited area with high signal intensity in the region of the right cerebellar peduncle, without enhancement after contrast administration. There is an extensive area of gliosis located to the cortex of the left fronto-parietal region of the cerebral hemisphere, with associated enlargement (ex vacuo) of the body, the frontal and temporal horn of the ipsilateral lateral ventricle (Figure $4 \mathrm{~A}-\mathrm{C}$ ) (the patient exhibits a right-sided hemiparesis, with hypertonia of her left side with increased deep tendon reflexes ipsilaterally).

The patient underwent serial repeated MRI examinations, in order to monitor the possible development of additional pathological features, but the only differentiating finding with respect to the previous images was the visualization of atrophic changes in the domain of the right cerebral peduncle, without enhancement after administration of contrast medium. The final imaging was performed 3 years after the initial radiological evaluation, which verified the aforementioned findings (Figure 5A-D).

EEG revealed a basic rhythm of $\theta$ waves (5-6c/sec) (discharges with rhythmic frequency) when the patient was waking, which could represent an ictal onset pattern. During all the recording period, we recognize the very frequent occurrence of paroxysmal discharges of spike-multispike wave form and of focal sharp waves that are mainly recorded from the scalp electrodes covering the fronto-temporal regions of the left cerebral hemisphere. These tend to propagate from the generating source to the relevant regions of the contralateral hemisphere and become generalized (generalized discharges). It is worthwhile to mention that spikes in the waking state (or during active sleep) provide the best localizing data.

\section{Discussion And Conclusions}

There have been several published adult cases of brain tumor initially misdiagnosed as viral encephalitis $[6,7]$.This is exactly the opposite, compared to our patient, in whom the final diagnosis was HSV infection. Very few cases of Herpes Simplex Encephalitis resembling clinical and imaging features of central nervous system tumor have been described in the literature [1].

The diagnosis of HSE can be confounded with other clinical entities because of the presence of misinterpreted or borderline clinical, laboratory and imaging data. Interpreting these results can be further clouded by the fact that our patient was afebrile during the first days of his hospital course, which is uncommon in HSE. Besides, temporal lobe involvement is classical for herpes encephalitis, in contrast 
with the cerebellum, especially when it is the only involved area, as in our patient, at least in the initial phase of his disease.

MR spectroscopy results in some patients are inadequate to verify a definitive diagnosis. This was the case in our patient, as there was a discrepancy between the definitive diagnosis and the one proposed by the aforementioned examination. Relevant MR spectroscopy measurements regarding brain neoplastic lessions include decreased $\mathrm{N}$-acetylaspartate (NAA), which is a marker indicative of neuronal cell damage, increased choline (Cho), indicating cell membrane and myelin reconstruction, and decreased creatine ( $\mathrm{Cr}$ ), which is a pool of inorganic phosphates, used for ATP production, and eventually a marker of the available molecules utilized for energy production. Non-neoplastic disease entities referring to CNS, comprising infections, may cause as a consequence an active response of the immune system, which can include reactive proliferation of various cell types, and, additionally, of glial elements. This sequence of events can result in the production of specific MR spectroscopy patterns that are difficult to differentiate the analogous profiles of CNS neoplasms, as both types of offending lessions share in common their tendency to result in neuronal cell damage and detriment [8].

MR spectroscopy findings in HSE patients commonly consist of decreased NAA/Cr fraction, measured at different and not discrete time points during the disease process, which could be interpreted as indicative of neuronal cell death. Another well described measurement is an increased Cho/Cr fraction, which may be representative of myelin damage and disintegration $[9,10]$.

Our case illustrates the concerns of definitive diagnosis in some patients with a clouded clinical picture, even if all the appropriately indicated workup (including bloodwork, imaging, and MR spectroscopy) was undertaken. When confounding results occur, we believe that tissue diagnosis (stereotactic or open biopsy) is essential to ensure appropriate diagnosis and disease management. In this case, although biopsy via craniotomy was performed because of concerns that we were confronted with a cerebellar glioma requiring surgical excision, the histopathologic and immunohistochemical analysis did not declare a definite diagnosis (of note that we had obtained tissue samples twice.

When we are confronted with such diagnostic and therapeutic dilemmas, it is wise to keep in our mind the remote possibility that the results of all the aforementioned tests may be confounding. We cannot underestimate the importance of the evolving clinical picture of the patient. The cerebellar lesion was diagnosed incidentally in an asymptomatic period and therefore it was very unlikely to represent smoldering HSV1 encephalitis, which typically has a much more fulminant course. In our case, the patient became febrile a few days after the operation and even later epileptic activity was added to her clinical picture, obtaining a clinical and neurological picture resembling encephalitis. Our last diagnostic tool, except from brain biopsy, was CSF examination, as it was done in our case (PCR assay for HSV DNA). As MRS and MRI results may be inefficient to distinguish a brain tumor from a viral infection, CSF examination may provide the definitive diagnosis and guide our treatment regimen.

\section{Declaration}


- Ethics approval and consent to participate: A local ethics committee ruled that no formal ethics approval was required in this particular case. We confirm that the consent we obtained from study participants (namely, from the parents of our patient) was written and it could be available to you, upon reasonable request.

- Consent for publication: We confirm in this statement that written consent to publish this information was obtained from the parents of our patient. We are be advised that proof of consent to publish from parents can be requested at any time and it would be available to your Editorial Team.

- Availability of data and materials:

- The datasets used and/or analysed during the current study are available from the corresponding author on reasonable request.

- All data generated or analysed during this study are included in this published article [and its supplementary information files].

- Competing interests: The authors declare that they have no competing interests.

- Funding: Not Applicable.

- Authors' contributions:

- DP, IT, KA conceived and designed the analysis of the case description. They made also substantial contributions to conception and design, and acquisition of data, and

analysis and interpretation of data.

- PK, GS, MT participated in drafting the article and revising it critically for important intellectual content.

- DP, IT, KA, PK, GS, MT gave final approval of the version to be submitted and any revised version.

Each author should have participated sufficiently in the work to take public responsibility for appropriate portions of the content. Allowing one's name to appear as an author without having contributed significantly to the study or adding the name of an individual who has not contributed or who has not agreed to the work in its current form is considered a breech of appropriate authorship.

- Acknowledgments: Not Applicable.

- Formatting of funding sources: This research did not receive any specific grant from funding agencies in the public, commercial, or not-for-profit sectors

\section{List Of Abbreviation}

HSV: Herpes Simplex Virus 
MRI: Magnetic Resonance Imaging

MRS: Magnetic Resonance Spectroscopy

CSF: Cerebrospinal Fluid

LHRH: Luteinizing Hormone Releasing Hormone

NAA: N-Acetyl Aspartate

Cr: Creatine

Cho: Choline

GFAP: Glial Fibrillary Acidic Protein

NeuN: Neuron specific Nuclear Antigen

T2W: T2 Weighted

FLAIR: Fluid Attenuation Inversion Recovery

T1 FFE CM: T1 Fast Field Echo Contrast Medium

T2 TSE: T2 Turbo Spin Echo

ICU: Intensive Care Unit

CT: Computed Tomography.

EEG: Electroencephalography

MIB-1: Methylation-Inhibited Binding Protein

Real Time qRT-PCR Real-Time Quantitative Reverse Transcription Polymerase Chain Reaction

DNA: Deoxyribonucleic Acid

RNA: Ribonucleic Acid

FRET: Fluorescence resonance energy transfer-based real-time polymerase chain reaction method without DNA extraction

UL28= Infected cell protein 18.5 (ICP18.5)

HCMV: Human Cytomegalovirus

ATP: Adenosine triphosphate 


\section{Reference List}

1. Peeraully T, Landolfi JC. Herpes Encephalitis Masquerading as Tumor. ISRN Neurology. 2011; Article ID 474672, 4 pages. doi:10.5402/2011/474672.

2. Whitley R.J, Lakeman F. Herpes simplex virus infections of the central nervous system: therapeutic and diagnostic considerations. Clinical Infectious Diseases 1995; 20(2):414-420.

3. Whitley R.J, Soong S.J, Linneman Jr C, et al. Herpes simplex encephalitis: clinical assessment. Journal of the American Medical Association 1982; 247(3):317-320.

4. J. H. Rees and R. S. Howard, "High-grade glioma mimicking acute viral encephalitis-three case reports. Postgraduate Medical Journal 1999;75 (890): 728-730.

5. Weil A. A., Glaser C. A, Amad Z, et al. Patients with suspected herpes simplex encephalitis: rethinking an initial negative polymerase chain reaction result. Clinical Infectious Diseases 2002;34 (8):1154-1157.

6. Anaplastic astrocytoma mimicking herpes simplex encephalitis in 13-year old girl. Talathi S, Gupta N, Reddivalla N, et al. European Journal of Paediatric Neurology 2015;19:722-725. http://dx.doi.org/10.1016/j.ejpn.2015.07.007.

7. Wang J, Luo B. Glioblastoma masquerading as herpes simplex encephalitis. Journal of the Formosan Medical Association 2015;114:1295-1296.

8. Krouwer H. G. J, Kim T. A, Rand S. D. et al. Singlevoxel proton MR spectroscopy of nonneoplastic brain lesions suggestive of a neoplasm. American Journal of Neuroradiology. 1998; 19 (9):1695-1703.

9. Takanashi J. I, Sugita K, Ishii M, et al. Longitudinal MR imaging and proton MR spectroscopy in herpes simplex encephalitis. Journal of the Neurological Sciences 1997; (149) 1: 99-102.

10. Hitosugi M, Ichijo M, Matsuoka Y, et al. Proton MR spectroscopy findings in herpes simplex Encephalitis. Clinical Neurology 1996; 36(7): 839-843.

\section{Figures}




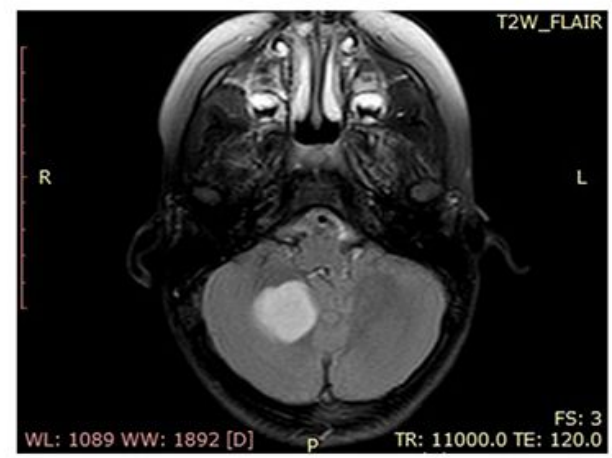

Figure 1.A

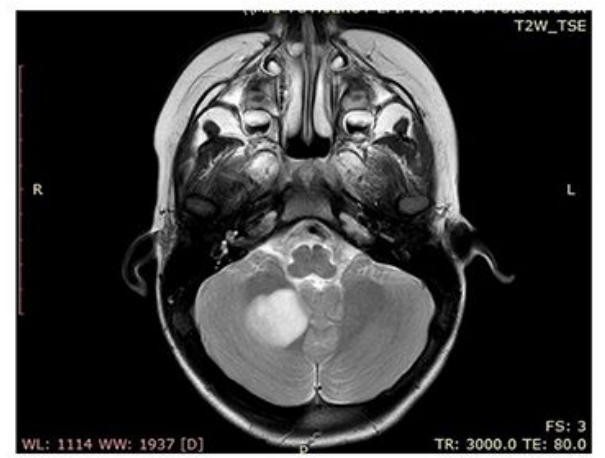

Figure 1.B

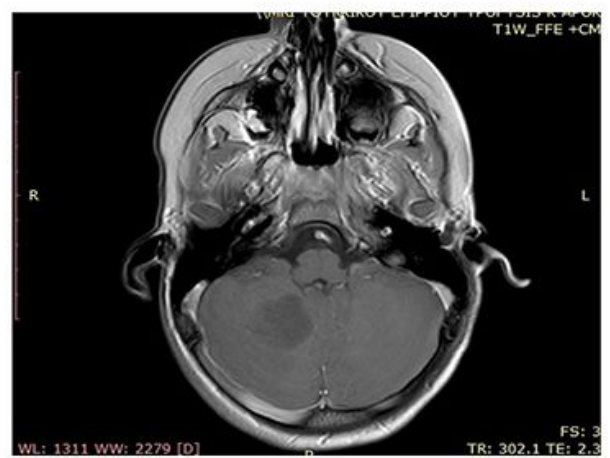

Figure 1.C

\section{Figure 1}

A: Initial MRI scan, axial FLAIR image, demonstrating the right cerebellar lesion without significant surrounding edema. And no midline shift. B: Axial T2 TSE scan of the initial MRI, showing similar results as the previous scan. C: Initial MRI, axial T1 FFE scan, after intravenous administration of paramagnetic substance, verifying the absence of contrast enhancement from the mass lesion. 


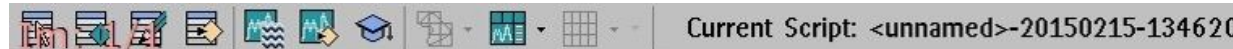
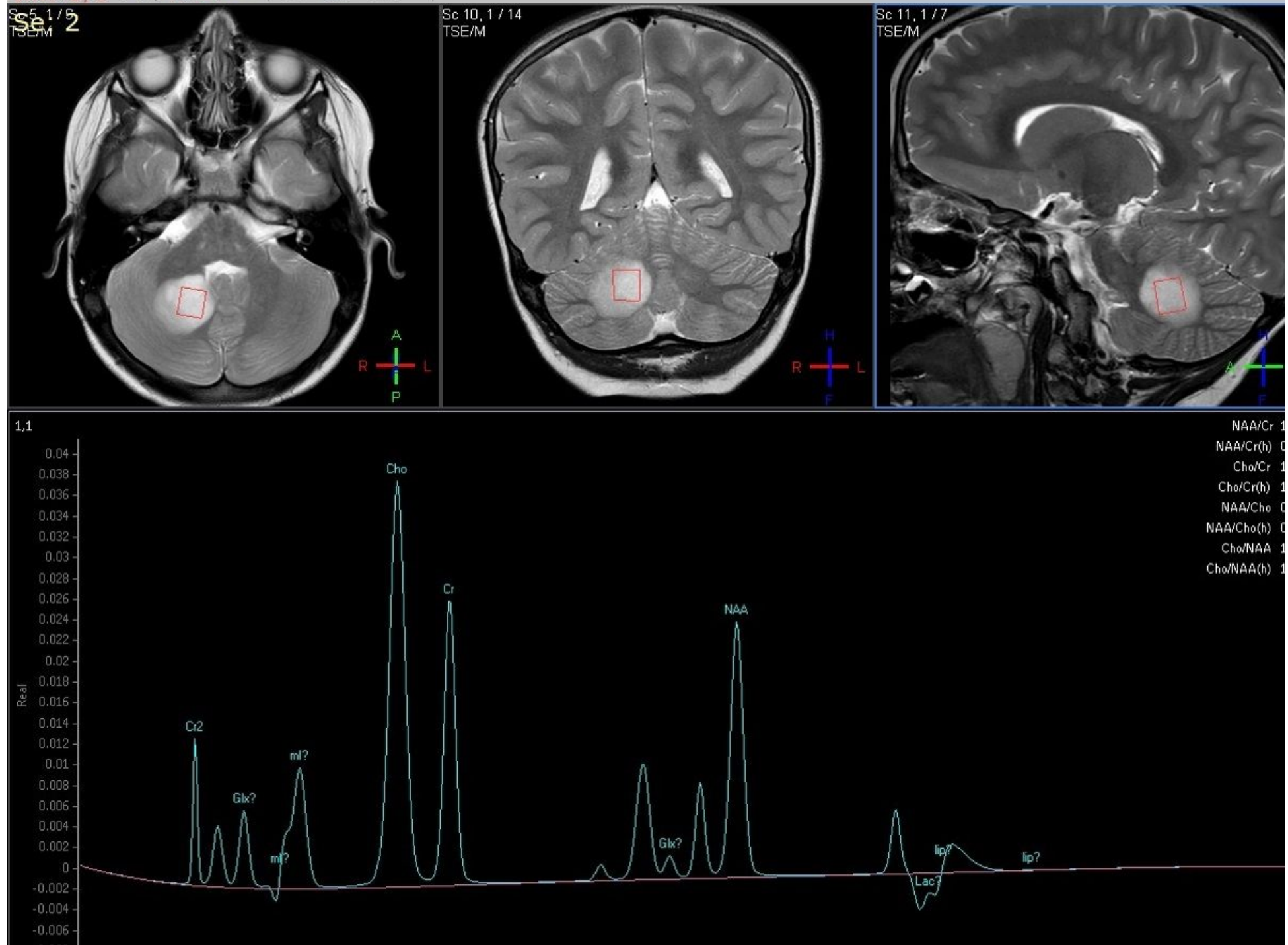

$\mathrm{NAA} / \mathrm{Cr}$ $\mathrm{NAA} / \mathrm{Cr}(\mathrm{h})$ $\mathrm{Cho} / \mathrm{Cr}$ $\mathrm{Cho} / \mathrm{Cr}(\mathrm{h})$ NAA/Cho NAA/Cho(h) Cho/NAA Cho/NAA(h)

$\begin{array}{lllllllllllllllllllllll}4.3 & 4.2 & 4.1 & 4 & 3.9 & 3.8 & 3.7 & 3.6 & 3.5 & 3.4 & 3.3 & 3.2 & 3.1 & 3 & 2.9 & 2.8 & 2.7 & 2.6 & 2.5 & 2.4 & 2.3 & 2.2\end{array}$

\section{Spectro Results}

\begin{tabular}{|c|c|c|c|c|c|c|c|c|c|c|c|c|c|c|c|}
\hline Metab & Position & SNR & Width & Height & $\mathrm{Ht} / \mathrm{Cr}$ & Area & $\mathrm{Ar} / \mathrm{Cr}$ & Metab & Position & SNR & Width & Height & $\mathrm{Ht} / \mathrm{Cr}$ & Area & $\mathrm{Ar} / \mathrm{Cr}$ \\
\hline NAA & 2.008 & 3.9 & 0.051 & 0.025 & 0.888 & 0.001 & 0.997 & $\mathrm{Cr}$ & 3.031 & 4.3 & 0.045 & 0.028 & 1.000 & 0.001 & 1.000 \\
\hline W/ cho- & $8.4 \mathrm{M}$ & 1.67 & 50.19 & 0.039 & 1.406 & 0.003 & 1.938 & $m I ?$ & $3561 ?$ & $1.8 ?$ & $0051 ?$ & $0,012 ?$ & $0,414 ?$ & $0,001 ?$ & $0.466 ?$ \\
\hline VV $L_{m I}$ & 9.62 & $13 !$ & $0.05 ?$ & $0.008 ?$ & $0.300 ?$ & $0,000 ?$ & $0.337 ?$ & $\mathrm{Cr} 2$ & 3.934 & 2.4 & 0.017 & 0.015 & 0.544 & 0.000 & 0.207 \\
\hline Glx? & $3.853 ?$ & a9? & $0.037 ?$ & $0.006 ?$ & $0.211 ?$ & $0,000 ?$ & $0.373 ?$ & $G 6 x^{2}$ & $3760 ?$ & $12 ?$ & $0,037 ?$ & $0,007 ?$ & $0265 ?$ & $0000 ?$ & $0.217 ?$ \\
\hline G/x? & 36392 & -2.22 & $0037 ?$ & $-0,008 ?$ & $-0.284 ?$ & 0,0002 & -0.2332 & atk? & 24922 & 0,22 & $0,037 ?$ & 00002 & $0,054 ?$ & 00002 & 0044 \\
\hline
\end{tabular}

\section{Figure 2}

MRS, demonstrating the peaks of NAA, Cho and $\mathrm{Cr}$. 


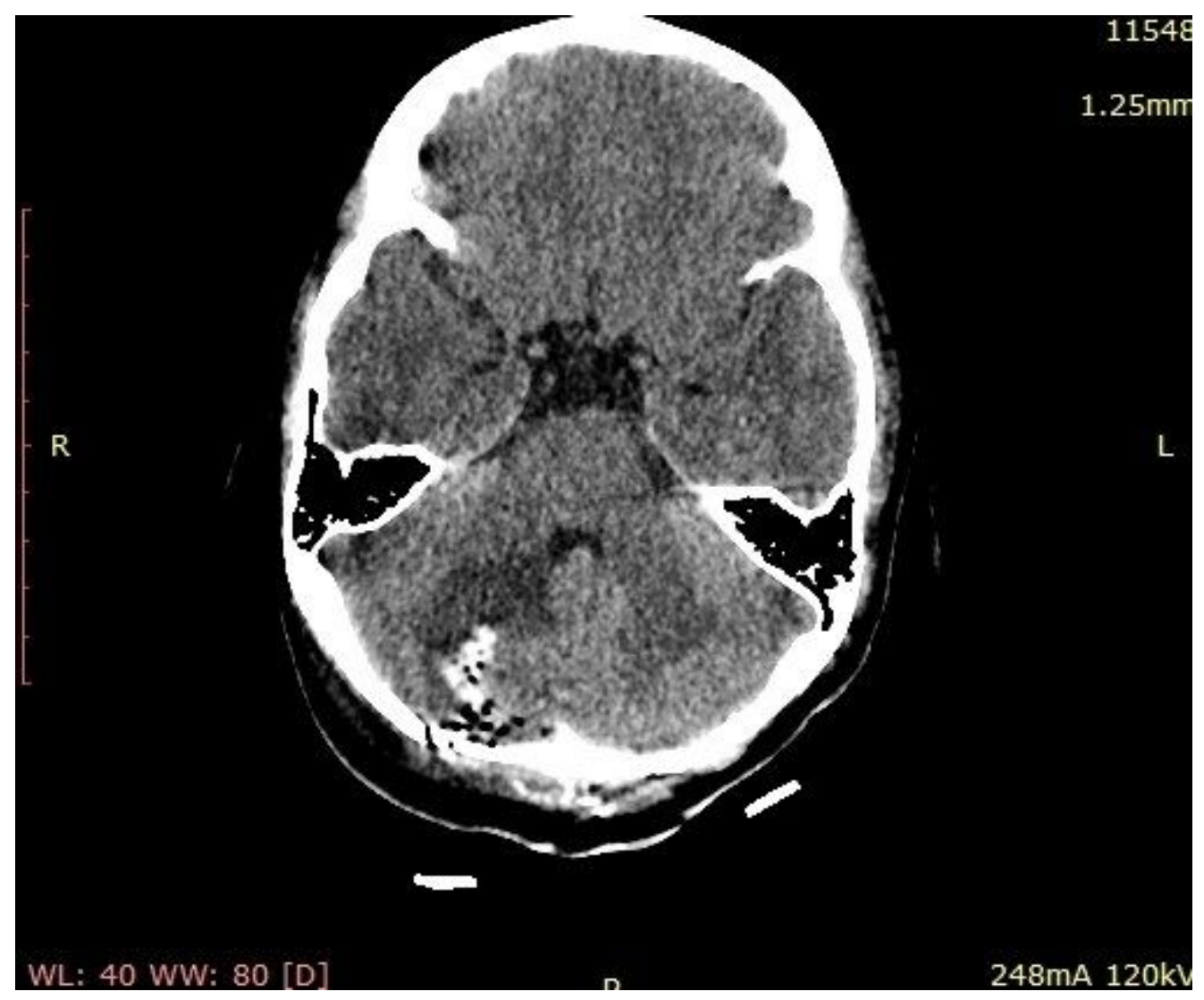

\section{Figure 3}

Immediate post-operative CT scan, demonstrating a well-circumscribed hypodense lesion remain in the resection bed, giving the impression of residual lesion.

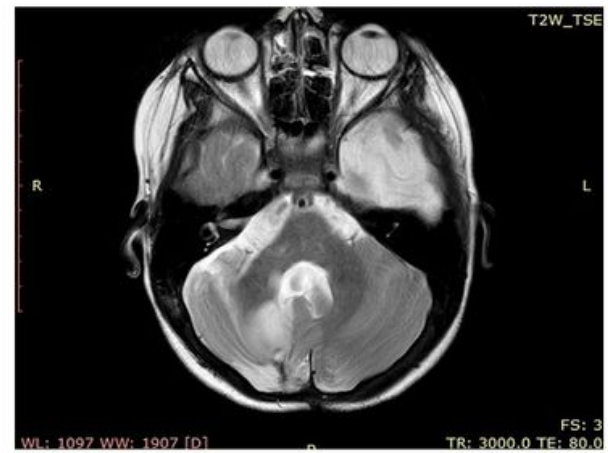

Figure 4.A

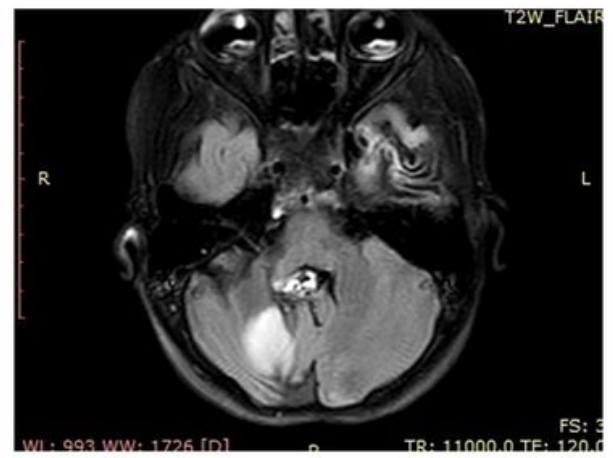

Figure 4.B

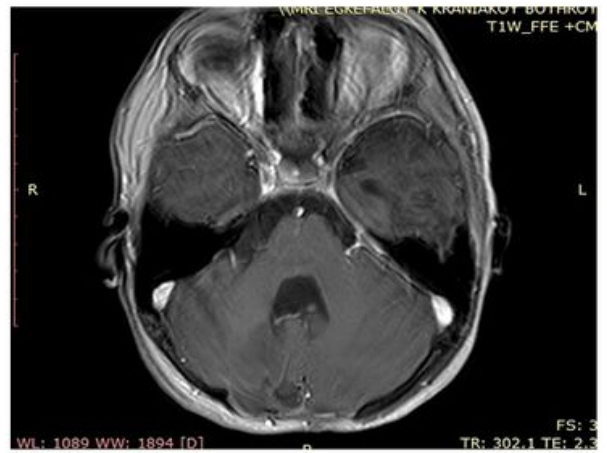

Figure 4.C

\section{Figure 4}

A: MRI scan six months later, axial T2 TSE scan, identifying a region on the right cerebellar hemisphere with pathologic high signal intensity. B: MRI scan six months later, axial T2 FLAIR scan, with similar pathologic imaging as the previous scan. C: MRI scan six months later, axial T1 FFE scan after intravenous administration of paramagnetic substance, showing no pathologic uptake from the offending mass lesion. 


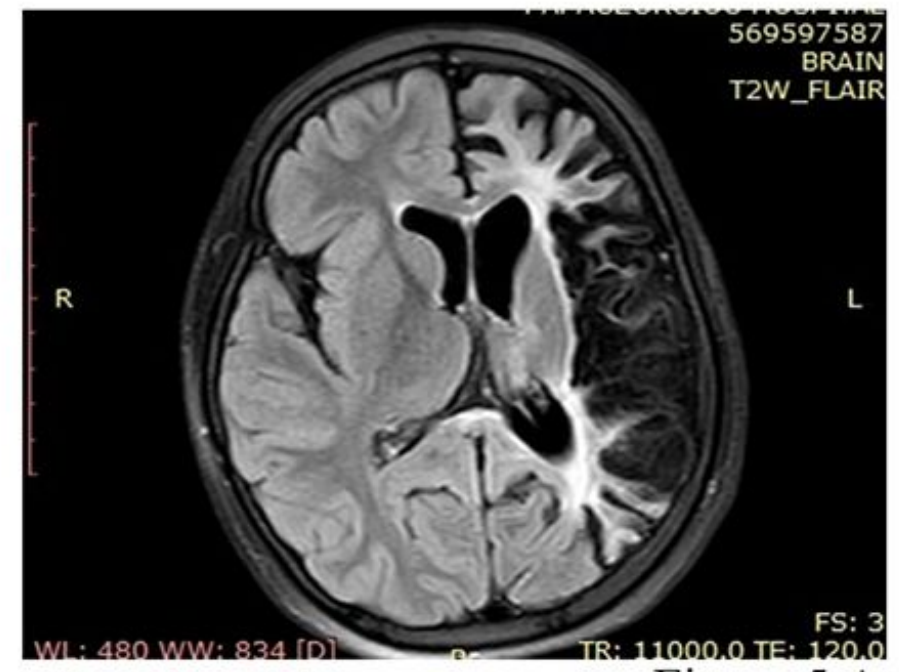

Figure 5.A

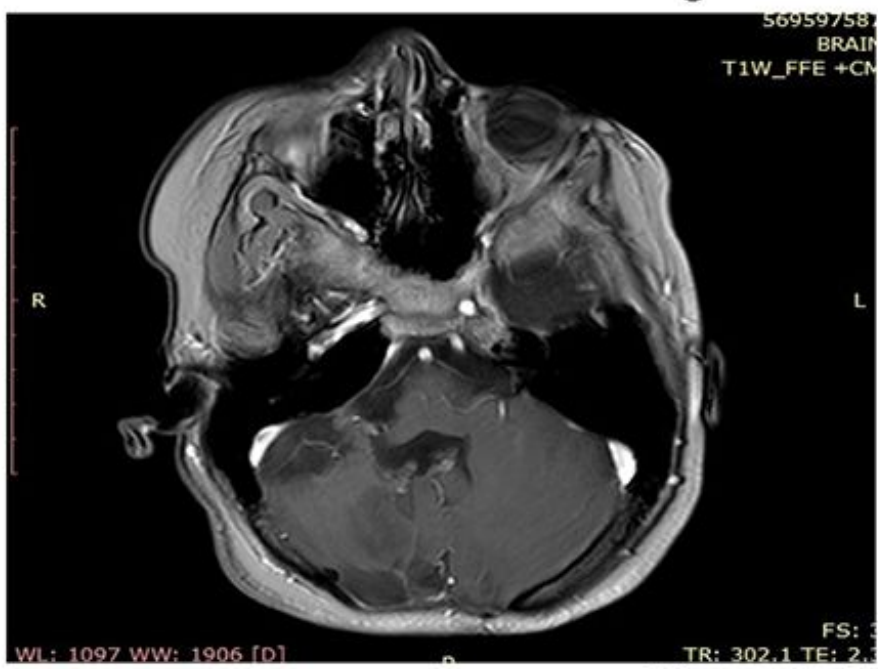

Figure 5.C

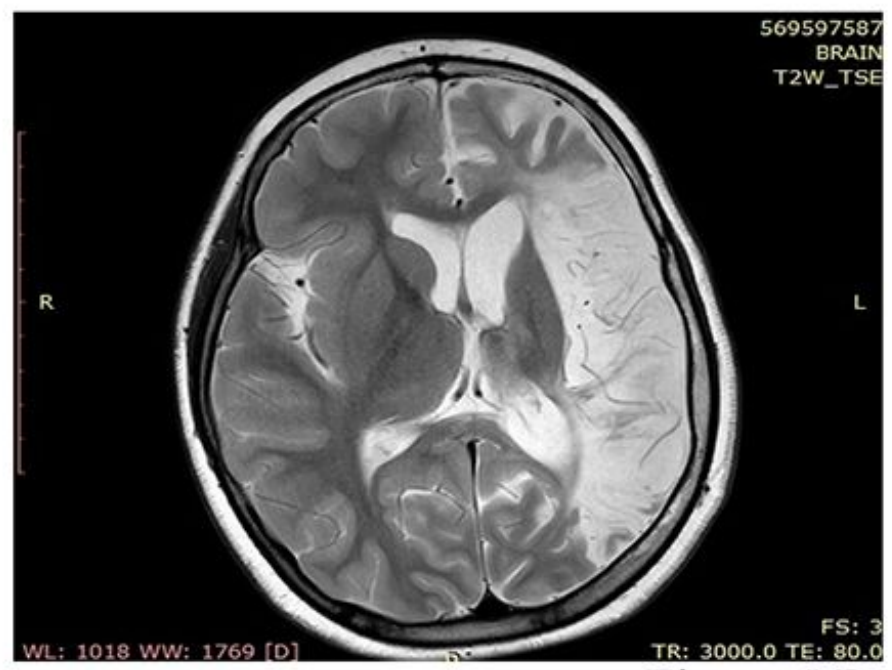

Figure 5.B

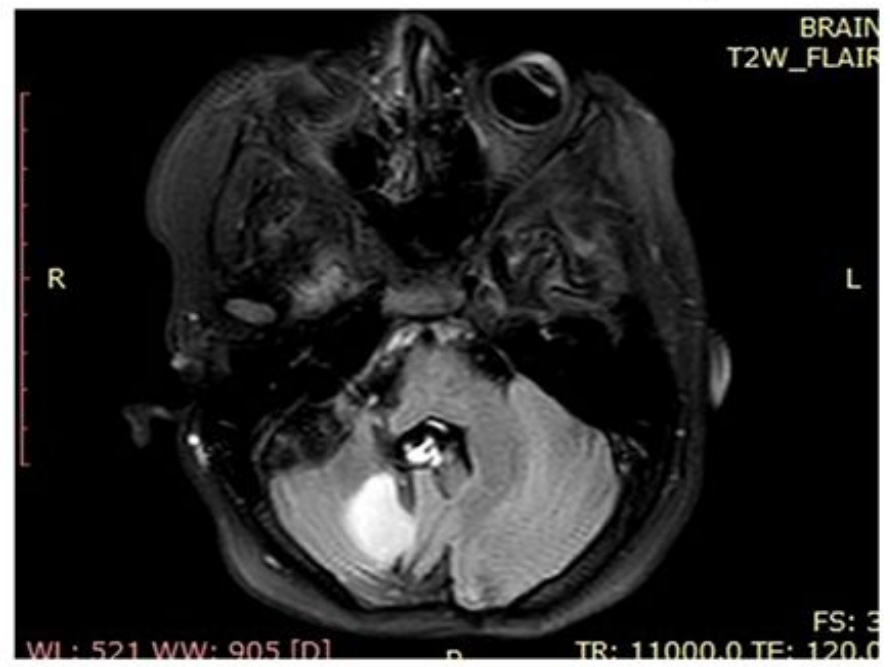

Figure 5.D

\section{Figure 5}

A: MRI scan three years after the initial imaging, axial T2 FLAIR, showing the extensive gliomatous changes in the region of the left cerebral hemisphere. B: MRI scan three years after the initial imaging, axial T2 TSE scan, delineating similar pathologic changes with the previous scan. C: MRI scan three years after the initial imaging, axial T1 FFE scan after intravenous administration of paramagnetic substance, showing no contrast uptake from the offending lesion of the posterior fossa. D: MRI scan three years after the initial imaging, axial T2 FLAIR, demonstrating that the imaging characteristics of the initially diagnosed lesion of the posterior fossa do not demonstrate any significant differentiation.

\section{Supplementary Files}

This is a list of supplementary files associated with this preprint. Click to download.

- supplement1.pdf 